\title{
Investigation of the status quo of massive blood transfusion in China
}

\author{
Jiangcun Yang ${ }^{1^{*}}$, Qiushi Wang ${ }^{2}$, Yang Sun ${ }^{1}$, Ting Ma ${ }^{1}$, Shuming Zhao ${ }^{3}$ Bijuan $\mathrm{Li}^{4}$, \\ Cuixiang Xu ${ }^{1}$, Hang Chen ${ }^{1}$, Ziyun Shi ${ }^{1}$, Jing Liu ${ }^{5 *}$ \\ ${ }^{1}$ Department of Transfusion Medicine, Shaanxi Provincial People's Hospital, Xi'an, Shaanxi 710068, China; \\ ${ }^{2}$ Department of Transfusion Medicine, Shengjing Hospital of China Medical University, Shenyang, Liaoning 110004, China; \\ ${ }^{3}$ Department of Transfusion Medicine, Southwest Hospital, the Third Military Medical University, Chongqing 400038, China; \\ ${ }^{4}$ Department of Transfusion Medicine, Xiangya Hospital Center of South University, Changsha, Hunan 410013, China; \\ ${ }^{5}$ Division of Transfusion Medicine, Johns Hopkins University, School of Medicine, Baltimore, MD 21287, USA.
}

\begin{abstract}
The aim of this study was to provide an overview of massive transfusion in Chinese hospitals, identify the important indications for massive transfusion and corrective therapies based on clinical evidences and supporting experimental studies, and propose guidelines for the management of massive transfusion. This multi-region, multi-center retrospective study involved a Massive Blood Transfusion Coordination Group composed of 50 clinical experts specializing in blood transfusion, cardiac surgery, anesthesiology, obstetrics, general surgery, and medical statistics from 20 tertiary general hospitals across five regions in China. Data were collected for all patients, who received $\geqslant 10 \mathrm{U}$ red blood cell transfusion within 24 hours in the participating hospitals from January 12009 to December 31 2010, including patient's demographics, pre-, peri-, and post-operative clinical characteristics, laboratory test results before, during, and after transfusion, and patient mortality at post-transfusion and discharge. We also designed an in vitro hemodilution model to investigate the changes of blood coagulation indices during massive transfusion and the correction of coagulopathy through supplement blood components under different hemodilutions. The experimental data in combination with the clinical evidences were used to determine the optimal proportion and timing for blood component supplementation during massive transfusion. Based on the findings from the present study, together with an extensive review of domestic and international transfusionrelated literature and consensus feedback from the 50 experts, we drafted the guidelines on massive blood transfusion that may help Chinese hospitals to develop standardized protocols for massive blood transfusion.
\end{abstract}

Keywords: massive blood transfusion, Chinese, coagulopathy correction, hemodilution, guidelines, massive blood transfusion

\section{INTRODUCTION}

Blood transfusion plays an important life-saving role for patients with severe conditions and poten-

*Correspondence to: Dr. Jiang-Cun Yang, Department of Transfusion Medicine, Shaanxi Provincial People's Hospital, 256 Youyi West Road, Xi'an 710068, China. TEL: +86-029-85253261-3073; Fax: +86-029-85236987; E-mail: yjc65@sina.com; Jing Liu, Ph.D., Di- tially fatal conditions. Massive blood transfusion is defined as a transfusion of more than one entire blood volume in 24 hours $(\mathrm{h})^{[1-4]}$, replacement of $50 \%$ of total blood volume within $3 \mathrm{~h}^{[3,4]}$, or administration

vision of Transfusion Medicine, Johns Hopkins University, School of Medicine, $600 \mathrm{~N}$ Wolfe Street, Baltimore, MD 21287, USA; Email: jliu84@jhmi.edu. 
of $\geqslant 10$ units $(\mathrm{U})$ of red blood cell suspension (RBCS) to a patient within $24 \mathrm{~h}^{[5]}$. As an important transfusion therapy in response to massive and uncontrolled hemorrhaging, massive transfusion is often associated with high mortality and high coagulopathy leading to a high incidence of multi-organ failure and mortality especially in trauma patients ${ }^{[6-8]}$. To reduce the incidence of dilutional complications resulting from large volumes of RBCS transfusion and lower the risk of mortality, many countries and regions have developed guidelines on massive blood transfusion ${ }^{[3,4,9]}$. Timely implementation of institutional massive transfusion protocols has significantly improved the resuscitation efficiency and patient outcomes ${ }^{[10-13]}$. However, currently in China, there are no guidelines on massive transfusion, nor consensus on the critical indications and the optimal timing and ratio of blood components delivery in the correction of coagulopathy. As a matter of fact, little is known about the current blood transfusion practice in response to massive hemorrhage in Chinese hospitals.

According to the Protocols of Clinical Transfusion Management issued by the Chinese Ministry of Health in $2012^{[14]}$, a request of $800-1,600 \mathrm{ml}$ transfusion within $24 \mathrm{~h}$ needs approval of department directors; a request of $>1,600 \mathrm{ml}$ transfusion must be submitted by department directors and approved by the hospital Office of Medical Affairs, with the exception of emergency transfusion. Yet the Protocols provide neither a definition nor management guidelines for massive transfusion. With no clear definition of the indications for coagulopathy during massive transfusion and recommended corrective approaches, Chinese clinicians have to administer blood transfusions either based on their own judgment or by referring to foreign guidelines. Active bleeding occurs frequently during massive blood transfusions, which seriously affects the efficacy and outcome of emergency resuscitation.

This study was aimed to accomplish the following goals: first, to understand the current practice of massive transfusion and patient outcomes in China by examining the demographic and clinical characteristics, pre and post-transfusion blood test results, volumes and ratios of transfused blood components, and outcomes of patients who received $\geqslant 10 \mathrm{U}$ RBCS within $24 \mathrm{~h}$ of hospitalization. Second, to identify important indications for massive transfusion used by Chinese clinicians and evaluate the variations in timing and ratio of blood components delivery in the correction of coagulopathy, the clinical features of coagulopathy, frequency of blood tests during massive transfusion, and their association with patient mortality. Additionally, to better understand the mechanism of coagulop- athy and effective correction strategies, we designed an in vitro model of coagulopathy with a corrective approach to simulate in vivo coagulation during severe blood loss and collected data from laboratory experiments to verify our findings derived from clinical data. Third, based on the in vitro findings as well as evidences of clinical transfusion practices in Chinese hospitals and international massive transfusion guidelines, propose a definition of massive transfusion that fits the Chinese adult patient population, determine the objectives of massive blood transfusion protocol, and describe the important indications and strategies for massive transfusion management for Chinese clinicians.

\section{MATERIALS AND METHODS}

\section{Clinical data collection}

This retrospective study included 50 experts in clinical blood transfusion, cardiac surgery, trauma, anesthesiology, obstetrics, general surgery, and biostatistics from 20 tertiary general hospitals in five geographic regions in China (northwest, southwest, central south, north, and northeast). The number of beds varied greatly from hospital to hospital ranging from 2,000-7,000 beds and 20,000-65,000 annual surgeries in each hospital. From the blood bank electronic database at each hospital, all adult patients ( $\geqslant$ 18 years old) received of $\geqslant 10 \mathrm{U}$ of RBCs within $24 \mathrm{~h}$ after admission during the period of Januaryl 2009 to December 302010 were identified and included as subjects. Additional information on patient preoperative characteristics, intraoperative information, and postoperative outcomes was extracted from either computerized or paper medical charts, or surgery or anesthesiology records. We designed the data collection form "Questionnaire on Massive Blood Transfusion" that consisted of more than 60 items in 4 sections including patient demographic characteristics; type and volume of blood components transfused; perioperative complications and clinical conditions within and after $24 \mathrm{~h}$ of the transfusion; surgical and transfusion events; the laboratory test results before, during, and after blood transfusion such as routine blood test (RBT), blood coagulation tests (BCT), liver function test, kidney function test, and arterial blood gas analysis; adverse events after massive blood transfusion, and discharge status. All available blood test results and transfusion information at the following time points were collected: before RBCs transfusion and when RBCs transfusions reached the following volumes: $2 \mathrm{U}, 4 \mathrm{U}, 6 \mathrm{U}, 8 \mathrm{U}, 10 \mathrm{U}, 12 \mathrm{U}, 14 \mathrm{U}$, 
16U, 18U, 20U, 22U, 24U, 26U, 28U, 30U, 40U. In general, IU RBCS (140-172 ml) was derived from $200 \mathrm{ml}$ whole blood; 1 U FFP was $100 \mathrm{ml}$; one bag of apheresis Platelets (PLT) was $10 \mathrm{U}$ (1 bag $=250-$ $300 \mathrm{ml})$; 1 U PLT concentrate suspension $(25 \sim 35 \mathrm{ml})$ was derived from $200 \mathrm{ml}$ whole blood. Dedicated data collection staff from each participating hospital were selected and trained to complete the questionnaires according to the study protocol.

All collected questionnaire data were sent to the Blood Transfusion Department of Shaanxi Provincial People's Hospital. Data completeness, quality, and validity were assessed by a panel of experts in our research group based on the predetermined inclusion and exclusion criteria. Patients who underwent trauma, cardiac surgery, obstetric or other general surgeries (including orthopedic, thoracic, general, urinary, hepatobiliary, and neurological surgery) were included in the study; whereas patients with coagulation disorders, hepatic failure due to medical causes, and coagulopathies were excluded. Details about the method, i.e. the questionnaire, laboratory equipment and medical devices and reagent utilization across all participating hospitals, as well as laboratory testing result validity, reliability, and overall data quality control have all been published ${ }^{[15-21]}$. This study was approved by the Institutional Review Board at all participating institutes.

\section{Establishment of an in vitro model of coagu- lopathy and its correction protocol}

Massive hemorrhage was simulated by an in vitro hemodilution model and a correction protocol for coagulopathy was developed. Blood samples $(26 \mathrm{ml})$ were collected in $3.8 \%$ sodium citrate from 24 healthy staff at Shaanxi Provincial People's Hospital, including 14 females and 10 males, 19-50 years of age, with no history of anemia, coagulation disorders, hemorrhage, or kidney disease. Females undergoing menstruation were also excluded. All RBCS, FFP, and PLT components used for correction of anemia and coagulopathy due to hemodilutions were processed according to the standard protocol ${ }^{[20]}$. The blood samples of RBCS were prepared and stored for 3-5 days. FFP samples were defrosted, reading at $37{ }^{\circ} \mathrm{C}$ on the day of testing and apheresis PLT samples were obtained from the same day phlebotomy. Under diverse conditions: (saline dilution ratios 10:0, 9:1, 8:2, 7:3, 6:4, 5:5, 4:6, 3:7, 8:2 and 1:9 for control group; 7:3, 6:4, $5: 5,4: 6,3: 7,8: 2$ and $1: 9$ for the case group) indirectly reflecting the in vivo hemodilutional conditions of patients during massive transfusion, specific quantities of blood components including RBCs, platelets, and plasma were replenished to correct coagulopathy and anemia by maintaining the hemoglobin level at 60-80 g/L. A thromboelastograph (TEG) hemostasis analyzer (TEG 5000; Haemoscope Corp., Niles, IL, USA), automated blood coagulation analyzer (CA7000; Sysmex Corp., Kobe, Japan), and Fully Automatic Haemacytometer Analyzer (LH 750; Beckman Coulter Fullerton, CA, USA) were used for blood testing. Blood indices including RBC, hematocrit, and PLT count prior to and following the supplement of blood components and changes in the blood coagulation indices and coagulopathy were investigated. The hemostatic ability of infused RBCs and PLT at different dilution ratios were also evaluated in comparison to similar clinical data obtained from the multi-center study ${ }^{[18,20]}$.

\section{Development of massive transfusion guide- lines}

Based on the clinical data derived from our multicenter study and the in vitro experimental results, as well as extensive review of domestic and international transfusion-related literature, laws, and regulations, and consultation with more than 50 experts, we developed the guidelines for massive blood transfusion for adult patients $\geqslant 18$ years of age, especially those in general surgery, cardiac surgery, obstetric surgery, and trauma patients ${ }^{[22]}$.

\section{Statistical analysis}

All eligible clinical and experimental data were entered into the EPIDATA database (version 3.01; Epidata Association). Double entry method was used to ensure data accuracy. PASW Statistics for Windows version 18.0 (released 2009; SPSS Inc., Chicago, IL, USA) was used for statistical analysis. The data on the demographic characteristics and clinical features were reported as the mean \pm standard deviation or as counts and ranges. Categorical variables were analyzed by Chi-square statistics; continuous variables with normal distribution were analyzed by the Shapiro-Wilk test, analysis of variance or the KruskalWallis test, as appropriate. Linear regression or logistic regression analysis was performed to describe the association between continuous or dichotomous outcomes and covariates. A two-sided $P<0.05$ was set as the statistically significant level.

\section{RESULTS}

\section{Summary of clinical data from 20 hospitals}

A total of 2,000 surveys were distributed to 20 hospitals and 1,753 (87.6\%) completed questionnaires 
were collected, from which 1,601 (91.3\%) met all inclusion criteria. Among them, $553(34.5 \%)$ received $<10 \mathrm{U}$ RBCS and $1,048(65.5 \%)$ received $\geqslant 10 \mathrm{U}$ of RBCs within $24 \mathrm{~h}$ of hospitalization. All data reported in the present study were based on the 1,048 patients.

Patient demographics, clinical characteristics, preand post-transfusion blood test results, and mortal- ity for the 1,048 patients by clinical departments are displayed in Table 1. About half of these patients $(51.6 \%)$ were from General Surgery, $25.5 \%$ Cardiac Surgery, $17.8 \%$ Trauma, and $5.1 \%$ Obstetrics. More male $(n=646,61.6 \%)$ than female patients $(n=402$, $38.4 \%$ ) were included. Patients in Obstetrics (mean age $=33 \mathrm{yrs}$ ) and Trauma (mean age $=40 \mathrm{yrs}$ ) were

Table 1 Demographic and clinical characteristics, pre-transfusion blood indices, and summary oftotal transfused components for all patients

\begin{tabular}{|c|c|c|c|c|c|}
\hline & Trauma & Cardiac surgery & General surgery & Obstetrics & P-values \\
\hline Number of patients, $n$ ( $\%$ of total) & $187(17.8)$ & $267(25.5)$ & $541(51.6)$ & $53(5.1)$ & $<0.001$ \\
\hline Males, $n(\%)^{*}$ & $138(73.8)$ & $169(63.3)$ & $339(62.7)$ & $0(0.0)$ & $<0.001$ \\
\hline Female, $n(\%)$ & $49(26.2)$ & $98(36.7)$ & $202(37.3)$ & $53(100)$ & $<0.001$ \\
\hline Age $(y r$, mean $\pm S D)$ & $40( \pm 15)$ & $46( \pm 17)$ & $47( \pm 17)$ & $33( \pm 7)$ & $<0.001$ \\
\hline Weight $(\mathrm{kg}$, mean $\pm \mathrm{SD})$ & $60( \pm 10)$ & $57( \pm 13)$ & $59( \pm 11)$ & $60( \pm 7)$ & $<0.001$ \\
\hline Operation time $(\mathrm{h}$, mean $\pm \mathrm{SD})$ & $3.47( \pm 4.38)$ & $5.33( \pm 3.87)$ & $3.18( \pm 3.70)$ & $1.78( \pm 2.52)$ & $<0.001$ \\
\hline Length of ICU stay (d, mean \pm SD) & $9( \pm 12)$ & $7( \pm 9)$ & $13( \pm 43)$ & $3( \pm 4)$ & 0.917 \\
\hline Length of hospital stay (d, mean $\pm S D$ ) & $31( \pm 28)$ & $32( \pm 23)$ & $30( \pm 24)$ & $18( \pm 8)$ & 0.353 \\
\hline Males, $n(\%$ out of total male $)$ & $23(16.7)$ & $34(20.1)$ & $14(4.1)$ & 0 & $<0.001$ \\
\hline Female, $n(\%$ out of total female $)$ & $4(8.2)$ & $15(15.3)$ & $15(7.4)$ & $3(5.7)$ & 0.112 \\
\hline \multicolumn{6}{|l|}{ Before transfusion } \\
\hline $\mathrm{RBC} \times 10^{12} / \mathrm{L},($ mean $\pm \mathrm{SD})$ & $3.3( \pm 1.0)$ & $4.3( \pm 1.0)$ & $3.8( \pm 1.0)$ & $3.2( \pm 1.0)$ & $<0.001$ \\
\hline $\mathrm{Hb}$ in $\mathrm{g} / \mathrm{L},($ mean $\pm \mathrm{SD})$ & $108( \pm 76)$ & $132( \pm 31)$ & $115( \pm 31)$ & $95( \pm 31)$ & $<0.001$ \\
\hline Hct as $\%,($ mean $\pm \mathrm{SD})$ & $16( \pm 17)$ & $13( \pm 18)$ & $19( \pm 17)$ & $11( \pm 14)$ & $<0.001$ \\
\hline $\mathrm{PLT} \times 10^{9} / \mathrm{L},($ mean $\pm \mathrm{SD})$ & $170( \pm 103)$ & $145( \pm 67)$ & $194( \pm 108)$ & $161( \pm 77)$ & $<0.001$ \\
\hline $\mathrm{PT}$ in $\mathrm{s},($ mean $\pm \mathrm{SD})$ & $16.9( \pm 11.1)$ & $13.9( \pm 3.8)$ & $13.5( \pm 4.1)$ & $14.6( \pm 6.6)$ & $<0.001$ \\
\hline APTT in $s,($ mean $\pm S D)$ & $41( \pm 29)$ & $34( \pm 9)$ & $35( \pm 18)$ & $47( \pm 66)$ & 0.009 \\
\hline $\mathrm{INR},($ mean $\pm \mathrm{SD})$ & $1.2( \pm 1.0)$ & $1.3( \pm 0.1)$ & $1.1( \pm 1.0)$ & $2.2( \pm 3)$ & 0.039 \\
\hline $\mathrm{FIB},($ mean $\pm \mathrm{SD})$ & $13.4( \pm 1.4)$ & $15.5( \pm 3.2)$ & $14.8( \pm 5.1)$ & $13.7( \pm 3.5)$ & 0.087 \\
\hline \multicolumn{6}{|l|}{ During transfusion } \\
\hline RBCs, median U in $24 / 72 \mathrm{hr}$ & $13 / 19$ & $13 / 17$ & $14 / 17$ & $14 / 20$ & $0.306 / 0.147$ \\
\hline FFP, median U in $24 / 72 \mathrm{hr}$ & $7 / 10$ & $18 / 22$ & $9 / 11$ & $10 / 12$ & $<0.001 /<0.001$ \\
\hline PLT, median U in 24/72 hr & $5 / 8$ & $3 / 3$ & $5 / 9$ & $3 / 4$ & $0.621 / 0.493$ \\
\hline Total RBCs Units Transfused & 3496 & 4179 & 7528 & 1081 & $<0.001$ \\
\hline Average \# of RBCs Unit per patient & 19 & 16 & 14 & 20 & $<0.001$ \\
\hline Total FFP Units Transfused & 1859 & 3151 & 4554 & 590 & $<0.001$ \\
\hline Average \# of FFP Unit per patient & 10 & 12 & 8 & 11 & $<0.001$ \\
\hline Number of people using $\operatorname{FFP}(\mathrm{N}, \%)$ & $113(60.4)$ & $225(84.3)$ & $381(70.4)$ & $40(75.5)$ & 0.149 \\
\hline Average FFP:RBCs ratio & 1:1.88 & $1: 1.33$ & $1: 1.65$ & $1: 1.83$ & 0.062 \\
\hline
\end{tabular}

*Denominators for all percentages were total number of patients in each department unless otherwise described. 
younger than those in Cardiac Surgery (mean age $=$ $46 \mathrm{yrs}$ ) and General Surgery (mean age $=47 \mathrm{yrs}$ ). The average weight of all patients was about $60 \mathrm{~kg}$, with considerable variations by gender and across departments. The average operation time and number of days in ICU care also varied across departments. The number of days in ICU of General urgery displays a very large variance (mean $\pm \mathrm{SD}=13 \pm 43$ days), indicating the heterogeneity of clinical setting. No significant differences in the LOS were found between Cardiac Surgery, General Surgery, and Trauma, all being significantly longer than the LOS in Obstetrics.

There were significant differences in most pretransfusion blood and coagulation indices between the four departments $\left(P^{\prime} s<0.05\right)$. Mean pre-operative hemoglobin levels varied from $95 \mathrm{~g} / \mathrm{L}$ in Obstetrics to $132 \mathrm{~g} / \mathrm{L}$ in Cardiac Surgery. Mean INR was $>1.5$ in Obstetrics but less than 1.5 in the other three departments. On average, patients in Obstetrics and Trauma received more RBCS transfusions (20 and $19 \mathrm{U}$ per patient, respectively) than patients in Cardiac Surgery and General Surgery (16 and 14 U per patient, respectively). Patients in Cardiac Surgery, Obstetrics, Trauma, and General Surgery on average received 12, 11,10 , and $8 \mathrm{U}$ of FFP, respectively.

The average mortality rate was $10.3 \%$, and the mortality rate in four departments is as follows: 49 cases in Cardiac Surgery (18.4\%), 27 cases in Trauma (14.4\%), 29 cases in General Surgery (5.4\%), and 3 cases in Obstetrics (5.7\%).

\section{Blood component supplement}

All hospitals administered FFP to supplement RBCS transfusion at various time points. About 20\% of patients, mostly in Cardiac Surgery, received FFP supplement when RBCS transfusion was initiated. After $\geqslant 6 \mathrm{U}$ RBCS were transfused, about half of the patients $(54.2 \%)$ were infused with FFP. When RBCS transfusion reached $\geqslant 10 \mathrm{U}, 72.4 \%$ of patients were given FFP supplement. Table 2 presents the number of patients who received FFP transfusion by RBCS units. In a separate analysis, we found that transfusion of FFP supplement also varied by departments. For example, plasma was often used in combination with RBCS at earlier stage of cardiac surgery operation and the RBCS:FFP ratio was maintained 1 to 1 throughout transfusion. However, in other departments, plasma was rarely used when RBCS infusion was less than 4 U. After $\geqslant 10 \mathrm{U}$ RBCS were transfused, almost all patients from the four departments received plasma supplement at the RBCs:FFP ratio of 1.5:1. These data were summarized in previous publications by our group ${ }^{[16,21]}$.

Due to the limited availability and accessibility in China, PLT and cryoprecipitate (CRYO) were transfused much less frequently and their dosages did not increase proportionally with the increase of RBCS transfusion. Fig. 1 presents the number of patients with FFP, PLT, and CRYO supplement at different stages of RBCS transfusion. Within $24 \mathrm{~h}$, when RBCS transfusion reached $10 \mathrm{U}, 20 \mathrm{U}$, and $30 \mathrm{U}$, $72.4 \%, 78.3 \%$, and $80.6 \%$ patients were provided with FFP supplement, respectively. However, only $4.4 \%$, $6.7 \%$, and $13.7 \%$ patients were given PLT and $16.4 \%$, $24.2 \%$, and $24.2 \%$ patients were given CRYO, respectively. The median number of RBCS, FFP, PLT, and CRYO units transfused within $24 \mathrm{~h}$ was $25,20,6$, and 5 respectively. Only three patients in Obstetrics with low PLT counts had PLT infusion at the initiation of RBCS transfusion. An increase in PLT trans-

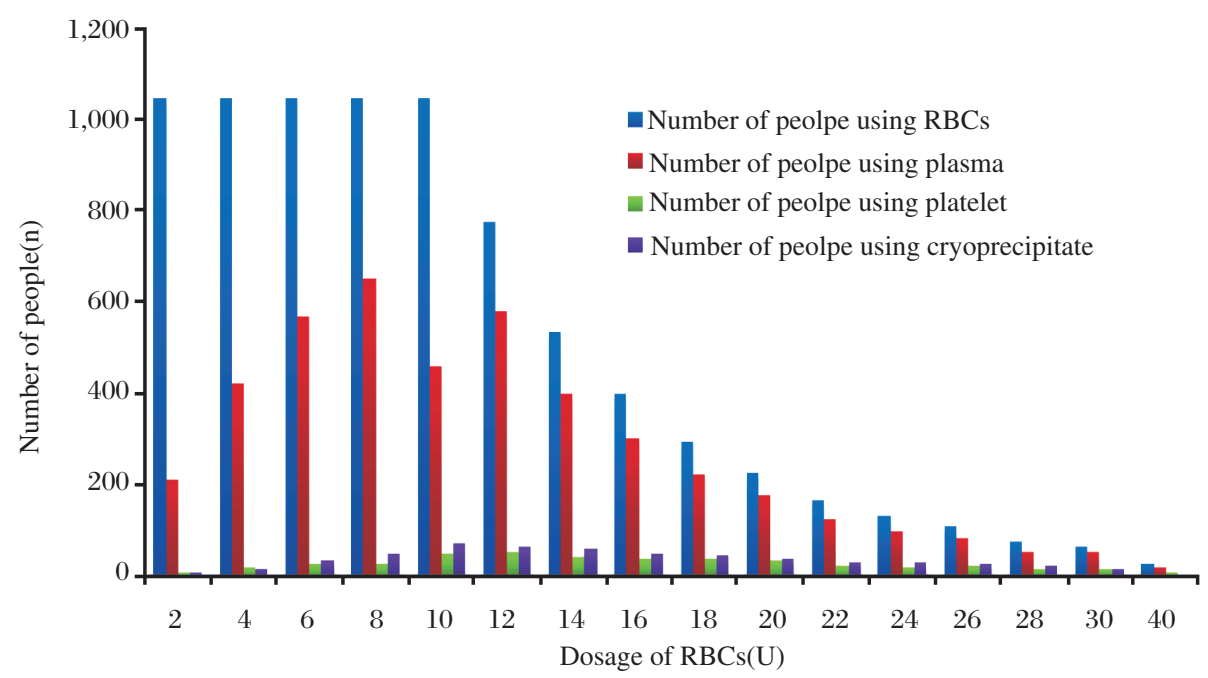

Fig. 1. Numbers of patients with RBCS transfusion and FFP, PLT, and CRYOsupplement under different RBCS dosages 
Table 2 Number of patients with FFP supplement and FFP dosages associated with different units of RBC

\begin{tabular}{|c|c|c|c|c|c|c|}
\hline \# of RBC Units & $\begin{array}{c}\text { Number of people using } \\
\operatorname{RBCS}(n)\end{array}$ & $\begin{array}{c}\text { Number of people } \\
\text { using } \operatorname{FFP}(n)\end{array}$ & $\begin{array}{c}\text { Ratio of number of people } \\
\text { using RBCs and FFP }\end{array}$ & RBCSdosage(U) & FFP dosage(U) & RBCS:FFP ratio \\
\hline 2 & 1,048 & 211 & 4.97 & 2,096 & 873.3 & 2.40 \\
\hline 4 & 1,048 & 422 & 2.48 & 4,192 & $2,164.5$ & 1.94 \\
\hline 6 & 1,048 & 568 & 1.85 & 6,288 & $3,607.5$ & 1.74 \\
\hline 8 & 1,048 & 651 & 1.61 & 8,384 & $5,027.7$ & 1.67 \\
\hline 10 & 1,048 & 759 & 1.38 & 10,480 & $6,796.8$ & 1.54 \\
\hline 14 & 535 & 398 & 1.34 & 7,462 & $5,099.1$ & 1.46 \\
\hline 16 & 400 & 301 & 1.34 & 6,368 & & 1.51 \\
\hline 18 & 293 & 222 & 1.32 & 5,238 & $3,475.7$ & 1.51 \\
\hline 20 & 226 & 177 & 1.28 & 4,480 & $3,021.4$ & 1.48 \\
\hline 22 & 166 & 124 & 1.34 & 3,608 & $2,230.7$ & 1.65 \\
\hline 24 & 131 & 95 & 1.38 & 3,096 & $1,874.8$ & 1.65 \\
\hline 28 & 76 & 53 & 1.43 & 2,072 & $1,133.3$ & 1.83 \\
\hline 30 & 62 & 50 & 1.24 & 1,800 & $1,159.5$ & 1.55 \\
\hline 40 & 26 & 19 & 1.37 & 960 & 449.5 & 2.14 \\
\hline Total & 1,048 & 759 & 1.38 & 16,284 & $10,154.6$ & 1.60 \\
\hline
\end{tabular}

fusion was found in a small number of trauma patients after they received 26-28 U RBCS, which might be random events. Similarly, an increase of CRYO infusion was observed only in Cardiac Surgery when transfused RBCS reached $30 \mathrm{U}^{[16]}$.

\section{RBCS dosage and mortality}

The association between the number of RBCS units transfused (RBCS dosage) and 30-day post-transfusion patient mortality was explored in the survival analysis. Fig. $2 \boldsymbol{A}$ and $\mathbf{2 B}$ display the Kaplan Meier survival plots for post-transfusion 30-day mortality rates grouped by different RBC dosages within 24 and 72 hours. Without adjusting for covariates, the survival rates significantly decreased (therefore mortality increased) as the volume of transfused RBCs increased from $10 \mathrm{U}$ to $40 \mathrm{U}$ within $24 \mathrm{~h}$ and $72 \mathrm{~h}$, $\left(P^{\prime} s<0.001\right)$. After adjusting for age, gender, weight, operation time, LOS, $24 \mathrm{~h}$ plasma dosage, and number

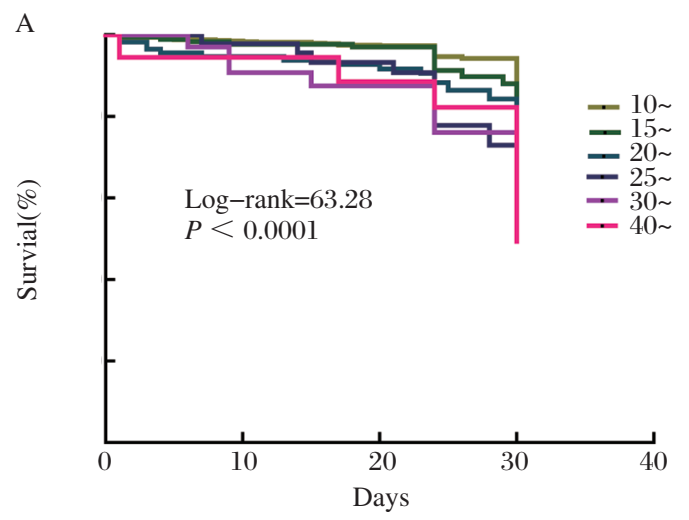

Kaplan Meier survival plot for mortality rates by different RBCs dosage in $24 \mathrm{~h}$ of days in ICU in logistic regression analysis, the volume of transfused RBCs used within $24 \mathrm{~h}$ was an independent risk factor for patient mortality, increasing infusion of every 2 RBCs units was associated with about 2-folds likelihood of mortality $(\mathrm{OR}=1.92$; 95\% CI: 1.56-2.33). Number of Days in ICU was also significantly associated with mortality, the longer the stay in ICU, the higher the mortality rate $(\mathrm{OR}=2.33$, 95\% CI: 1.14-4.76). Table 3 presents the results of the logistic regression analysis.

\section{FFP : RBCs ratio and mortality}

As shown in Table 2, up to $72.4 \%$ of the patients with RBCS transfusion also received plasma supplement at an average FFP: RBCS ratio of 1:1.5 when RBCs transfusion reached $\geqslant 10 \mathrm{U}$. To explore the potential impact of FFP: RBCS ratio and patient outcome, we divided the patients into three groups based on the $24 \mathrm{~h}$ and $72 \mathrm{~h}$ FFP: RBCS ratios sepa-

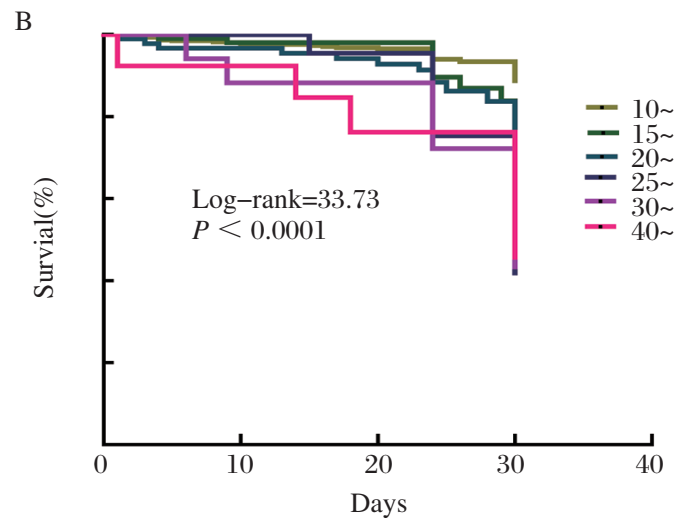

Kaplan Meier survival plot for mortality rates by different RBCs dosage in $72 \mathrm{~h}$

Fig. 2. Survival analysis on 30-day post-transfusion mortality rates by number of RBCs dosages transfused in 24 and 72 hours 
Table 3 Logistic regression analysis on mortality during admission

\begin{tabular}{cccc}
\hline & $\operatorname{Exp}(\mathrm{B})$ & \multicolumn{2}{c}{ Odds ratio(95.0\% CI) } \\
\hline 24h RBCSdosage (unit) & 1.92 & 1.56 & 2.33 \\
LOS(day) & 0.36 & 0.17 & 0.76 \\
Stay in ICU (day) & 2.33 & 1.14 & 4.76 \\
Age (year) & 1.01 & 0.99 & 1.03 \\
Gender & 1.82 & 0.83 & 4.00 \\
Weight (kg) & 1.02 & 0.99 & 1.05 \\
Operation time (hour) & 0.91 & 0.82 & 1.01 \\
24h FFP dosage (unit) & 1.00 & 0.96 & 1.03 \\
Intercept & 0.12 & & \\
\hline
\end{tabular}

rately: 1:0.3-0.75 was the high ratio group (HR); 1:1 2(1:0.75 2.3) was the medium ratio group (MR); $1: 2.5 \sim 4(1: 2.3 \sim 4)$ was the low ratio group (LR). There was no significant difference in the distribution of the three groups across the four clinical departments $(P=0.295)$. Fig. $3 \boldsymbol{A}$ and $\mathbf{3 B}$ display the 30-day Kaplan Meier survival plots for patients with different FFP: RBCS ratios during $24 \mathrm{~h}$ and $72 \mathrm{~h}$ transfusion, respectively. No significant differences in 30-day mortality rates were found among the 24 h FFP:RBCS ratio groups $(11.4 \%, 9.3 \%$, and $11.8 \%$ for HR, MR, and LR, respectively, $\chi^{2}=1.482, P=$ 0.197), although the medium ratio group maintained a slightly higher survival probability than the other two groups at 30-day post-transfusion. The advantage of medium $72 \mathrm{~h}$ FFP: RBCS ratio over high and low ratio on 30-day mortality was apparent, with a significantly lower mortality rate in the MR group (7.3\%) than in the HR (13.7\%) and LR (10.4\%) groups $\left(\chi^{2}=9.874\right), P<0.001$.

\section{Frequency of RBTs and BCTs during transfusion}

Overall, we obtained data from 1,013 (97\% of the $1,048)$ patients regarding blood testing during transfusion. No information on blood tests was retrieved for 35 patients. Among the 1,013 patients, $52.7 \%$ or 534 did not have any RBT during $24 \mathrm{~h}$ transfusion. About $27.8 \%, 13.6 \%, 4.2 \%$, and $1.6 \%$ of the 1,013 patients had $1,2,3$, and $\geqslant 4$ RBTs within 24 h during transfusion. For BCT, $79 \%$ or 800 patients did not have any test done, whereas $14.4 \%, 4.8 \%, 1.5 \%$, and $0.3 \%$ had $1,2,3$, and $\geqslant 4$ tests within $24 \mathrm{~h}$ of transfusion.

Table 4 presents the frequencies of RBTs or BCTs performed within $24 \mathrm{~h}$ during transfusion.

Table 4 Frequency of routine blood tests (RBTs) and blood coagulation tests (BCTs) within $24 \mathrm{~h}$ transfusion

\begin{tabular}{cccccc}
\hline & 0 & 1 & 3 & 3 & 4 \\
\hline RBTs & $534(52.7 \%)$ & $282(27.8 \%)$ & $138(13.6 \%)$ & $43(4.2 \%)$ & $16(1.6 \%)$ \\
BCTs & $800(79.0 \%)$ & $146(14.4 \%)$ & $49(4.8 \%)$ & $15(1.5 \%)$ & $3(0.3 \%)$ \\
\hline
\end{tabular}

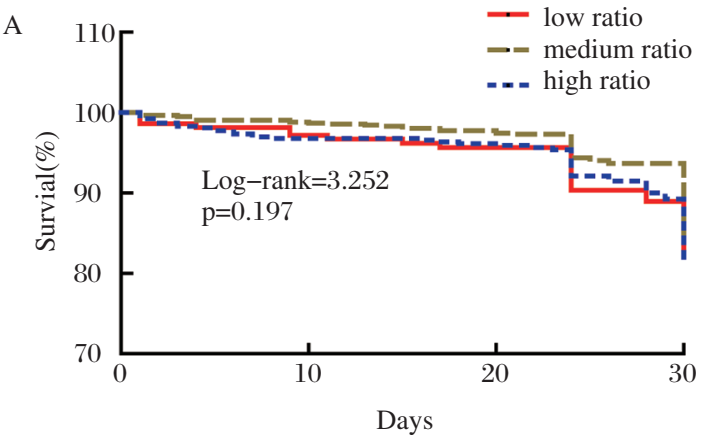

Kaplan Meier survival plot for 30-day mortality rates by different FFP:RBCs ratiosin $24 \mathrm{~h}$

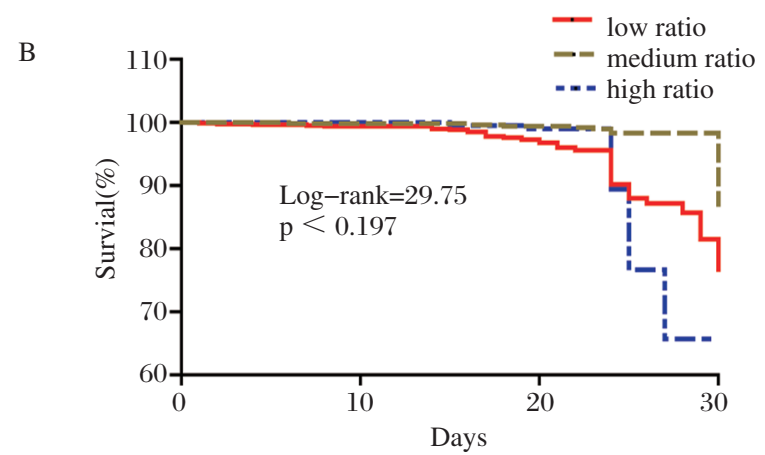

Kaplan Meier survival plot for 30-day mortality rates by different FFP:RBCs ratiosin $72 \mathrm{~h}$

Fig. 3. Survival analysis on 30-day post-transfusion mortality rates by transfused FFP:RBCs ratios in 24 and 72 hours

\section{Variations of PLT count during RBCs transfusion}

Among the 1,048 patients, 646 received RBC transfusion without PLT supplement and had at least one PLT count measured at one of the 16 time points during the first $24 \mathrm{~h}$ of transfusion. All PLT counts at the 16 predefined time points from these patients were selected and their association with the number of RBCS transfused was analyzed. One patient may contribute multiple PLT counts at various time points. When RBCS reached $18 \mathrm{U}$, the average PLT counts decreased to $72 \times 10^{9} / \mathrm{L}$. Similarly, when patients (those underweight with BMI < 18.5) were transfused with 0.3 units of RBCS per kilogram of body weight (i.e. $3 \mathrm{U} / 10 \mathrm{~kg}$ ), the average PLT count decreased to below $75 \times 10^{9} / \mathrm{L}$. Further laboratory testing found that the slight increase in the PLT count after RBC transfusion might be caused by the residual platelet like fragments in RBCs that were mistaken as PLTs by the machine. Regardless, a linear statistical regression model found a negative correlation between the number of RBCs units transfused and subsequent PLT count $\left(\mathrm{R}^{2}=0.641, P<.001\right)$. PLT count in these patients decreased with an increase in the number of RBCS units transfused. As shown in Fig. 4, the linear association was defined by the formula of $Y=127.3-$ 


\subsection{X.}

Based on these experimental findings and clinical evidence obtained from the 20 hospitals, we defined massive transfusion as a transfusion volume of RBCS

$18 \mathrm{U}$ within $24 \mathrm{~h}$, or $>=0.3 \mathrm{U} / \mathrm{kg}$ within $24 \mathrm{~h}^{[18,22]}$. According to this definition, among all the patients

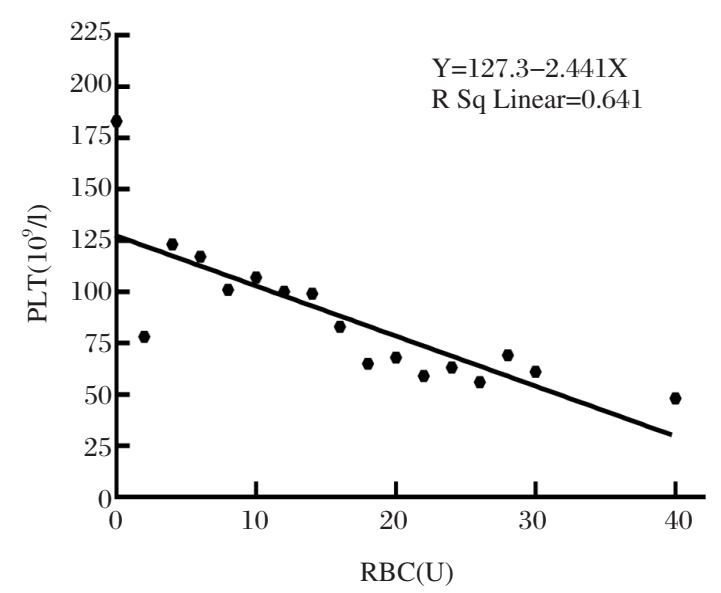

Fig. 4. Linear regression trend between units of RBCStransfused and PLT count among patients without PLT supplement during transfusion

with $\geqslant 10 \mathrm{U}$ RBCS within $24 \mathrm{~h}$ in our study, $28 \%$ received massive transfusion. A follow up analysis compared patient characteristics, clinical setting, pre-transfusion blood and coagulation indices, and transfused components between the two groups (Table 5). There were no significant differences in age, weight, clinical departments, pre-transfusion routine blood test and coagulation test results, ICU stay, and LOS between the two groups ( $\left.\mathrm{P}^{\prime} \mathrm{s}>0.05\right)$. However, patients with massive transfusion $(\geqslant 18 \mathrm{U}$ RBCs) received twice the amount of RBCS and FFP transfusion on average, had longer operation time (4.41 \pm 3.7 hours vs. $3.40 \pm 2.2$ hours) and higher overall mortality rate and at different FFP: RBCS ratios than those with < 18 U RBCS (18.8\% vs. 7.02\%), $\mathrm{P}^{\prime} \mathrm{s}<$ 0.001 . Females were more likely than males to receive massive transfusion $(39.3 \%$ vs. $20.9 \%, P<0.001)$.

\section{In vitro hemodilution experiments}

The PLT counts and coagulation indices prior to and at the 16 predefined time points after 2-40 units of RBCS transfusion were evaluated. Results showed that as the blood was increasingly diluted with RBCS transfusion, the dysfunction of coagulation increased with decreasing hemoglobin level, coagulation factor activity, and PLT count. The correction of the Hb level did not necessarily improve the coagulation indices. When the hemodilution levels reached $40 \%, 50 \%$, and $60 \%$, the hemoglobin level, coagulation indices, and
PLT count all reached critical levels, necessitating supplementation with RBCS (Hb, 60-80 g/L), FFP (FIB, $0.8-1.0 \mathrm{~g} / \mathrm{L}$; PT or APTT at N1.5 times the normal), and apheresis PLT (PLT count and TEG-MA, b40), respectively (More details about the experimental design and study methods can be found in previous reports by $\mathrm{Li}$ et al ${ }^{[18,20]}$ ). When hemodilution level was $90 \%$, the supplementation required for RBCS: FFP: PLT was approximately 1:1.3:0.9. The amount of supplement blood components to be administered increased proportionately with an increase of hemodilution, after the critical hemodilution level for each supplement was reached. Table 6 summarizes the guidelines to convert the in vitro proportions of RBCS, FFP, and PLT into vivo volumes. For example, for an average patient with a bodyweight of $60 \mathrm{~kg}$ and the total blood volume of $4,800 \mathrm{~mL}$, blood loss of $40 \%$ implies a loss of $1,920 \mathrm{~mL}$. Considering that the volume of $1 \mathrm{U}$ of RBCS is $140-172 \mathrm{~mL}(150 \mathrm{~mL}) ; 1$ $\mathrm{U}$ of FFP, $100 \mathrm{~mL}$; and $10 \mathrm{U}$ of PLT, about $250 \mathrm{~mL}$, the amount of RBCS supplementation required would be $6 \mathrm{U}$ of RBCS. Thus, when the blood loss is $70 \%$, or $3,360 \mathrm{~mL}$, the amount of component supplement required would be $16 \mathrm{U}$ RBCS, $19 \mathrm{U}$ FFP and $10 \mathrm{U}$ PLT, and the composition of the infusion required would be 1:1.2:0.6 for RBCS: FFP: PLT. However, when the amount of blood loss is the entire blood volume (dilution is $90 \%$, actual dilution is $96 \%$ ), the RBCS: FFP: PLT composition of the infusion required will be 1:1.3:0.9, and the amount of supplementation required would be $23 \mathrm{U}$ RBCS, $29 \mathrm{U}$ FFP and $20 \mathrm{U}$ PLT.

\section{Discussion}

The present study was a joint research project among 20 participating hospitals in China. The goal of this study was to evaluate the current practice of massive transfusion in China and provide evidence-based data and guidelines for the development of domestic massive transfusion protocols. The ultimate objectives were to standardize the procedure of massive transfusion management, help clinicians to stop bleeding and restore blood volume rapidly and efficiently in order to reduce the risks for adverse events associated with massive blood transfusion, and eventually improve the resuscitation success rate and patient outcomes. Efficient implementation of an effective massive transfusion protocol will also reduce potential waste of blood products, which has become a critical issue when the blood supply cannot meet the increasing request of clinical transfusion in Chinese hospitals ${ }^{[23,24]}$.

Our findings indicated that:

(1)During large volumes of RBCS transfusion, Chi- 
Table 5 Patient demographic and clinical characteristics, pre-transfusion blood indices, and summary of transfused components by total number of RBCs units transfused within 24 hours

\begin{tabular}{|c|c|c|c|}
\hline & \multicolumn{2}{|c|}{ RBCStransfused within $24 \mathrm{hr}$} & \multirow[b]{2}{*}{ P Value } \\
\hline & $10-17 \mathrm{U}$ & $>=18 \mathrm{U}$ & \\
\hline & $N=755,72 \%$ & $N=293,28 \%$ & \\
\hline Gender & & & $<0.001$ \\
\hline Males, $n(\%)$ & $511(67.7)$ & $135(46.1)$ & \\
\hline Female, $n(\%)$ & $244(32.3)$ & $158(53.9)$ & \\
\hline Age $(\mathrm{yr}$, mean $\pm \mathrm{SD})$ & $45 \pm 17$ & $45 \pm 11$ & 0.779 \\
\hline Weight $(\mathrm{kg}$, mean $\pm \mathrm{SD})$ & $59 \pm 12$ & $58 \pm 10$ & 0.444 \\
\hline Operation time (hour, mean $\pm \mathrm{SD}$ ) & $3.40( \pm 2.2)$ & $4.41( \pm 3.7)$ & $<0.001$ \\
\hline Length of ICU stay (day, mean \pm SD) & $9( \pm 10)$ & $9( \pm 7)$ & 0.996 \\
\hline Length of hospital stay (day, mean $\pm \mathrm{SD}$ ) & $29( \pm 22)$ & $31( \pm 19)$ & 0.177 \\
\hline Clinical Departments & & & 0.058 \\
\hline Trauma & 125 (16.6) & $62(21.1)$ & \\
\hline Cardiac surgery & $182(24.1)$ & $85(29.0)$ & \\
\hline General surgery & $409(54.2)$ & $132(45.1)$ & \\
\hline Obstetrics & $39(5.2)$ & $14(4.8)$ & \\
\hline \multicolumn{4}{|l|}{ Before transfusion } \\
\hline $\mathrm{RBC} \times 10^{12} / \mathrm{L},($ mean $\pm \mathrm{SD})$ & $3.9 \pm 1$ & $3.7 \pm 1.1$ & 0.345 \\
\hline $\mathrm{Hb}$ in $\mathrm{g} / \mathrm{L},($ mean $\pm \mathrm{SD})$ & $119 \pm 47$ & $114 \pm 32$ & 0.241 \\
\hline Hct as $\%,($ mean $\pm \mathrm{SD})$ & $17 \pm 18$ & $15 \pm 17$ & 0.075 \\
\hline $\mathrm{PLT} \times 10^{9} / \mathrm{L},($ mean $\pm \mathrm{SD})$ & $180 \pm 63$ & $165 \pm 88$ & 0.082 \\
\hline $\mathrm{PT}$ in $\mathrm{s},($ mean $\pm \mathrm{SD})$ & $14.1 \pm 6.2$ & $14.2 \pm 4.6$ & 0.546 \\
\hline APTT in $\mathrm{s},($ mean $\pm \mathrm{SD})$ & $36 \pm 25$ & $37 \pm 21$ & 0.674 \\
\hline $\mathrm{TT},($ mean $\pm \mathrm{SD})$ & $17 \pm 5.8$ & $18.9 \pm 9.8$ & 0.541 \\
\hline $\mathrm{INR},($ mean $\pm \mathrm{SD})$ & $1.2 \pm 0.6$ & $1.3 \pm 1.0$ & 0.145 \\
\hline $\mathrm{FIB},($ mean $\pm \mathrm{SD})$ & $11 \pm 4.3$ & $10.9 \pm 3.8$ & 0.097 \\
\hline \multicolumn{4}{|l|}{ During transfusion } \\
\hline RBCs, median U in $24 / 72 \mathrm{hr}$ & $12 / 15$ & $24 / 29$ & $<0.001 /<0.001$ \\
\hline FFP, median U in $24 / 72 \mathrm{hr}$ & $8 / 13$ & $14 / 16$ & $0.002 / 0.001$ \\
\hline PLT, median $\mathrm{U}$ in $24 / 72 \mathrm{hr}$ & $4 / 6$ & $8 / 9$ & $0.015 / 0.016$ \\
\hline Total RBCs Units Transfused & 9212 & 7072 & $<.001$ \\
\hline Average Number of RBCs Units per patient & 12 & 24 & $<.001$ \\
\hline Total FFP Units Transfused & 5967 & 4187 & $<.001$ \\
\hline Average Number of FFP Units per patient & 8 & 14 & $<.001$ \\
\hline Number of people using FFP(N, \%) & $537(71.1)$ & $222(75.8)$ & 0.117 \\
\hline Average FFP:RBCs ratio & $1: 1.54$ & $1: 1.69$ & 0.164 \\
\hline 24 h Mortality by FFP:RBCs ratio, n/total (\%) & & & $<0.001$ \\
\hline $1: 2.5 \sim 4$ & 11/121 (9.1) & 11/65 (16.9) & \\
\hline $1: 1 \sim 2$ & $24 / 419(5.7)$ & $31 / 172(18.0)$ & \\
\hline $2: 1$ & 17/215 (7.9) & $14 / 56(25.0)$ & \\
\hline $72 \mathrm{~h}$ Mortality by FFP:RBCs ratio, n/total (\%) & & & $<0.001$ \\
\hline $1: 2.5 \sim 4$ & $7 / 97(7.2)$ & 9/57 (15.8) & \\
\hline $1: 1 \sim 2$ & 12/333 (3.6) & $22 / 136(16.2)$ & \\
\hline $2: 1$ & $33 / 325(10.2)$ & $25 / 100(25.0)$ & \\
\hline Overall Mortality, N (\%) & $52(6.9 \%)$ & $56(19.1 \%)$ & $<0.001$ \\
\hline
\end{tabular}

nese clinicians focus on the supplement of FFP at early stages of RBCS transfusion. This practice is in accordance with the finding that balanced use of plasma early in resuscitation was associated with improved survival rates especially among trauma patients ${ }^{[25-28]}$. However, insufficient consideration is given to the use of PLT and cryoprecipitate ${ }^{[15,16]}$. According to AABB recommendation, early initiation of prophylactic PLT transfusion among adult patients with a PLT count of $10 \times 10^{9}$ cells/L or less may reduce the risk for spontaneous bleeding ${ }^{[29]}$. Cryoprecipitate and PLT transfusion were also reported to benefit the management of bleeding related to thrombolytic therapy ${ }^{[30]}$. However, due to the small number of patients who received PLT and CRYO transfusion, the small volumes of infusion, and the inconsistent RBCS: PLT: CRYO ratio and timing of infusion, we could not evaluate the effectiveness of these two components on patient outcomes in the present study. Future researches are needed to investigate the immediate and long term impact of PLT and cryoprecipitate supplement on Chinese patients with massive transfusion.

(2)Total units of RBCs transfused within $24 \mathrm{~h}$ were significantly associated with lower survival probabil- 
Table 6 Volumes of RBCS, FFP, and PLTsupplement by hemodilution levels during transfusion

\begin{tabular}{|c|c|c|c|c|c|c|}
\hline \multirow{2}{*}{$\begin{array}{l}\text { Hemodilution(add } \\
\text { RBCs :saline) }\end{array}$} & \multirow{2}{*}{$\begin{array}{l}\text { Actual Dilu- } \\
\text { tion }(\%)\end{array}$} & \multirow{2}{*}{$\begin{array}{l}4,800 \mathrm{ml} \text { Amount of blood loss } \\
\text { in } \mathrm{ml} \text { at a body weight of } 60 \mathrm{~kg}\end{array}$} & \multicolumn{4}{|c|}{$\begin{array}{l}\text { Amount of blood component supplement determined by in vitro tests and con- } \\
\text { verted into in vivo transfusion quantity }\end{array}$} \\
\hline & & & RBCS, $\mu \mathrm{L}$ & FFP, $\mu \mathrm{L}$ & PLT, $\mu \mathrm{L}$ & RBCS:FFP:PLT \\
\hline 1000:0 & 0 & 0 & & & & \\
\hline $900: 100$ & 10 & 480 & & & & \\
\hline 800:200 & 20 & 960 & & & & \\
\hline $700: 300$ & 36 & 1,440 & $100(480 \mathrm{ml} / 3 \mathrm{U})$ & & & \\
\hline 600:400 & 50 & 1,920 & $200(960 \mathrm{ml} / 6 \mathrm{U})$ & & & \\
\hline $500: 500$ & 66 & 2,400 & $300(1,440 \mathrm{ml} / 10 \mathrm{U})$ & $200(10 \mathrm{U})$ & & \\
\hline 400:600 & 77 & 2,880 & $400(1,920 \mathrm{ml} / 13 \mathrm{U})$ & $300(13 \mathrm{U})$ & $20(5 \mathrm{U})$ & 1:1:0.4 \\
\hline $300: 700$ & 85 & 3,360 & $500(2,400 \mathrm{ml} / 16 \mathrm{U})$ & $400(19 U)$ & $40(10 \mathrm{U})$ & 1:1.2:0.6 \\
\hline 200:800 & 91 & 3,840 & $600(2,880 \mathrm{ml} / 19 \mathrm{U})$ & $500(24 U)$ & $60(15 U)$ & 1:1.3:0.8 \\
\hline 100:900 & 96 & 4,320 & $700(3,360 \mathrm{ml} / 23 \mathrm{U})$ & $600(29 U)$ & $80(20 \mathrm{U})$ & 1:1.3:0.9 \\
\hline
\end{tabular}

ity, even after adjusting for potential confounding factors such as operation time and ICU stay that are critical indicators of disease severity. Those who received massive transfusion ( $\geqslant 18 \mathrm{RBCs} \mathrm{U}$ in $24 \mathrm{~h}$ ) displayed significantly higher mortality rate $(19.1 \%)$ than the other patients with $10-17 \mathrm{U}$ in $24 \mathrm{~h}(6.9 \%)$. Since no pre-transfusion differences were found between patients massive transfusion and those with $<18$ RBCs $\mathrm{U}$ in the first $24 \mathrm{~h}$, it is speculated that the higher patient mortality might be due to greater risks for respiratory and infectious complications associated with massive transfusion ${ }^{[31]}$, highlighting the importance of further investigation on the adverse events of massive transfusion and opportunities for improvement of massive transfusion management.

(3)The dilution and consumption of platelets and coagulation factors during massive transfusion often lead to coagulation complications. Therefore, early transfusion of FFP and PLT at appropriate ratios plays an important role in preventing coagulation disorders ${ }^{[18,21,26-28]}$. Our findings suggest that the amount of blood components replenished should increase proportionally to blood dilution levels at different time points, and that a medium FFP: RBCs ratio of 1:1.5 or the 1:1.3:0.9 RBCS: FFP: PLT ratio (based on in vitro experiments) is optimal to prevent common coagulation disorders and reduce fatality rate related to massive transfusion ${ }^{[19]}$. Our proposed ratios are the closest approximation to reconstituted whole blood and are in line with the 1:1:1 RBCS: FFP: PLT transfusion ratio recommendedby international literature ${ }^{[9-13]}$. However, our clinical data suggest that when the volume of FFP transfusion increased and was greater than that of RBCS within $24 \mathrm{~h}$ or $72 \mathrm{~h}$, an increase in patient mortality followed. Further investigation is needed to understand the mechanisms of moderate FFP: RBCS ratios at early stages of transfusion.
(4)The lack of laboratory monitoring of blood indices and coagulation factors during massive transfusion is consistent with a previous report in which among 35 surveyed hospitals in Sichuan (one of the most populated Chinese provinces) only $24.2 \%$ had systematic evaluations for the appropriateness and effect of blood component transfusion ${ }^{[32]}$. These findings underscore the great potential for improvement in clinical transfusion practice in China. Establishment of a standard massive transfusion protocol, in addition to the training and education of dedicated personnel for transfusion services and automated blood bank information systems as suggested by previous research ${ }^{[32]}$, are equally important to improve the efficacy of massive transfusion and optimize patient outcomes.

(5)Based on our clinical data on patients without PLT supplement within $24 \mathrm{~h}$ of transfusion and the in vitro experimental data on PLT variations, we defined massive blood transfusion as total transfusion volume of $\mathrm{RBCs} \geqslant 18 \mathrm{U}$ within $24 \mathrm{~h}$, or $\geqslant 0.3 \mathrm{U} / \mathrm{kg}$ within $24 \mathrm{~h}^{[18,22]}$. The rationale for this definition is consistent with the recommended PLT threshold of $75 \times 10^{9} / \mathrm{L}$ for trauma patients ${ }^{[33]}$. Follow up analysis comparing patient and clinical characteristics and pre-transfusion blood testing results between patients with 10-17 $\mathrm{U}$ RBCS and those with $\geqslant 18 \mathrm{U}$ RBCS within $24 \mathrm{~h}$ found very few differences at baseline but significant differences in post-transfusion outcomes, providing further evidence to support this demarcation.

(6)Based on laboratory data and findings from our evidence-based clinical practices in China and previous literature on massive transfusion in China and foreign countries, as well as a thorough review of relevant international and domestic laws and regulations on clinical transfusion, and feedback from more than 50 experts, our Massive Blood Transfusion Coordination Group drafted and finalized the massive 
transfusion guidelines and protocol after many revisions ${ }^{[22]}$. The guidelines is recommended for use in perioperative massive transfusion in general surgery, trauma, cardiac surgery, and obstetrics and excludes the treatment of blood loss caused by internal medical diseases, including blood coagulation disorders, liver failure, and other internal medical conditions.

\section{Acknowledgments}

This study was supported by a grant from Johnson (China) Medical Equipment Co., Ltd. We thank all the medical centers participating in this research: ShiJie Mu, Ai-Jun Xia and Xian-Qin Zhang from Xijing Hospital, the Fourth Military Medical University; Dai-Yu Li from Affiliated Hospital of Luzhou Medical College; Wei Jiao from the People's Hospital of Zhuang Autonomous Region; Li Tong from the First Affiliated Hospital of Kunming Medical University; Qing-Bao Meng from Shenzhen People's Hospital; Jie Li from the Fourth Clinical Medical College of Hebei Medical University; Shi-Ming Yang from Tangdu Hospital, the Fourth Military Medical University; Suo-Liang Yao from Xi'an Hong Hui Hospital; Cui-Ying Li from General Hospital of Chengdu Military Region; Mei-Ning Han from the Second Affiliated Hospital of Medical College of Xi'an Jiaotong University; Zhi-Xi Hu from Yan'an University Affiliated Hospital; Jin-Shan Jiao from the First Affiliated Hospital of Shanxi Medical University; Xian-Ping Lv from the First Affiliated Hospital of Zhengzhou University; Yan-Li Bai from Xi'an Central Hospital; Xiao-Xia Shi from Xianyang 215 Hospital; and FangXiang Chen from Daping Hospital, the Third Military Medical University.

\section{References}

[1] Malone DL, Hess JR, Fingerhut A. Massive transfusion practices around the globe and a suggestion for a common massive transfusion protocol. J Trauma, 2006; 60 (sup):S91-6.

[2] Schuster KM, Davis KA, Lui FY, et al. The status of massive transfusion protocols in United States trauma centers: massive transfusion or massive confusion? Transfusion, 2010, 50 (7):1545-1.

[3] Raymer JM, Flynn LM, Martin RF. Massive transfusion of blood in the surgical patient. Surg Clin North Am, 2012; 92:221-34

[4] Massive transfusion protocol (MTP) for hemorrhagic shock ASA committee on blood management. [Last accessed on 2014 Sep 27]. Available from: https://www. asahq.org/For-Members/About-ASA/ASA-Committees/Committee-on-Blood-Management.aspx [accessed 16.02.27].
[5] Vijaya Patil and Madhavi Shetmahajan. Massive transfusion and massive transfusion protocol. Indian J Anaesth, 2014; 58(5): 590-5. gov/pmc/articles/ PMC4260305/[accessed 16.02.27].

[6] Brohi K, Singh J, Heron M, Coats T. Acute traumatic coagulopathy. J Trauma, 2003; 54:1127.

[7] Maegele M, Lefering R, Yucel N, et al. Early coagulopathy in multiple injuries: an analysis from the German Trauma Registry on 8724 patients. Injury, 2007; 38: 298.

[8] Johansson PI, Stensballe J. Effect of haemostatic control resuscitation on mortality in massively bleeding patients: a before and after study. Vox Sang, 2009; 96: 111-8.

[9] The American Society of Anesthesiologists. Practice guidelines for perioperative blood transfusion and adjuvant therapies. Anesthesiology, 2015; 122: 241-5.

[10] Nunez TC, Dutton WD, May AK, Holcomb JB, Young PP, Cotton BA. Emergency department blood transfusion predicts early massive transfusion and early blood component requirement. Transfusio, 2010; 50(9):1914-20.

[11] Cotton BA, Gunter OL, Isbell J, et al. Damage control hematology: the impact of a trauma exsanguination protocol on survival and blood product utilization. J Trauma, 2008; 64(5): 1177-83.

[12] O’Keeffe T, Refaai M, Tchorz K, Forestner JE, Sarode R. A massive transfusion protocol to decrease blood component use and costs. Arch Surg, 2008; 143(7): 686-91.

[13] Riskin DJ, Tsai TC, Riskin L, et al. Massive transfusion protocols: the role of aggressive resuscitation versus product ratio in mortality reduction. J AmColl Surg, 2009; 209(2): 198-205.

[14] The Ministry of Health of People's Republic of China. Protocols of Clinical Transfusion Management in Medical Institutes. June 7, 2012. http://www.moh.gov. cn/mohzcfgs/s3576/201206/55072.shtml [accessed 16.02.27].

[15] Yang JC, Sun Y, Yan H, et al. Application status of blood constituents during massive blood transfusion in some regions of China. Int J Clin Exp Med, 2014; 7(7): 1775-80.

[16] Sun Y,Jin ZhK, Yang JC, et al. Investigation of the current situation of massive blood transfusion in different surgical departments: A large multicenter study in China. Int J Clin Exp Med, 2015; 8(6): 9257-65.

[17] Yang JC, Sun Y, Yan H, et al. Correlation between red blood cell transfusion volume and mortality in patients with massive blood transfusion: A large multicenter retrospective study. Exp Ther Med, 2015; 9:137-42.

[18] Yang JC, Sun Y, Yan H, et al. Coagulation Defects Associated with Massive Blood Transfusion: A Large Multicenter Study in China. Mol Med Rep, 2015; 12: 417986.

[19] Yang JC, Xu CX, Yan H, et al. Balanced ratio of plasma to packed red blood cells improves outcomes in massive transfusion. Exp Ther Med, 2015; 10: 37-42.

[20] Li L, Yang JC, Sun Y, et al. Correction of blood coagulation dysfunction and anemia by supplementation of red blood cell suspension, fresh frozen plasma, and apheresis 
platelet: Results of in vitro hemodilution experiments. J Crit Care, 2015; 30(1): 220.e1-e12.

[21] Jin ZhK, Sun Y, Yang JC, et al. The plasma and platelet are important in reducing the mortality in surgical massive blood transfusion: a large multicenter study in China. Int J Clin Exp Med, 2015,8(1):1073-9

[22] Yang JC, Xu YG, Sun Y, et al. The guidelines for massive blood transfusion. Chinese Journal of Blood Transfusion, 2012, 25(7): 617-621.

[23] China's "blood famine" drives patients to the black market. http://www.reuters.com/article/china-health-bloodidUSL4N0VN09020150214 [accessed 16.02.27].

[24] Yin YH, Li CQ, Liu Z. Blood donation in China: sustaining efforts and challenges in achieving safety and availability. Transfusion, 55, (10): 2523-30.

[25] Sperry JL, Ochoa JB, Gunn SR, et al. Inflammation the Host Response to Injury Investigators. An FFP:PRBC transfusion ratio $\& \mathrm{gt} ; /=1: 1.5$ is associated with a lower risk of mortality after massive transfusion. J Trauma, 2008; 65(5): 986-93.

[26] Gonzalez EA, Moore FA, Holcomb JB, et al. Fresh frozen plasma should be given earlier to patients requiring massive transfusion. J Trauma, 2007; 62(1): 112-9.

[27] Zink KA, Sambasivan CN, Holcomb JB, et al. A high ratio of plasma and platelets to packed red blood cells in the first 6 hours of massive transfusion improves outcomes in a large multicenter study. Am J Surg, 2009, 197(5): 565-70.

[28] Peralta R, Vijay A, El-Menyar A, et al. Trauma resuscitation requiring massive transfusion: a descriptive analysis of the role of ratio and time. World J Emerg Surg, 2015,;10: 36. eCollection 2015.

[29] Kaufman RM, Djulbegovic B, Gernsheimer T, et al. Platelet transfusion: a clinical practice guideline from the AABB. Ann Intern Med, 2015; 162(3): 205-13.

[30] Droubatchevskaia N, Wong MP, Chipperfield KM, et al. Guidelines for cryoprecipitate transfusion. BCMJ, 200749(8):441-5.

[31] Turan A, Yang D, Bonilla A, et al. Morbidity and mortality after massive transfusion in patients undergoing noncardiac surgery. Can J Anaesth, 2013; 60(8): 761-70.

[32] Liu Y, Lin J, Zhong L, et al. Blood transfusion practice: a survey in Sichuan, China. Transfus Apher Sci, 2015; 52(1):105-11.

[33] Stainsby D, MacLennan S, Hamilton PJ, et al. Management of massive blood loss: a template guideline. $\mathrm{Br} \mathrm{J}$ Anaesth, 2000, 85(3): 487-91.

(Received 23 December 2016, Revised 14 February 2017, Accepted 22 February 2017) 